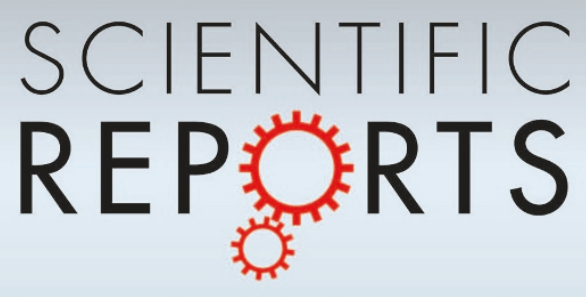

OPEN

SUBJECT AREAS:

OPTICAL SENSORS

TOTAL INTERNAL REFLECTION

MICROSCOPY

Received

16 January 2014

Accepted

23 June 2014

Published

10 July 2014

Correspondence and requests for materials should be addressed to Q.Y. lyeqing@nankai. edu.cn) or J.T. (ijitian@ nankai.edu.cn)

\section{Scanning focused refractive-index microscopy}

\author{
Teng-Qian Sun', Qing Ye', Xiao-Wan Wang', Jin Wang' ', Zhi-Chao Deng' ', Jian-Chun Mei², \\ Wen-Yuan Zhou' ', Chun-Ping Zhang' \& Jian-Guo Tian'
}

'Key Laboratory of Weak-Light Nonlinear Photonics, Ministry of Education, School of physics and TEDA Applied Physics School, Nankai University, Tianjin 300071, China, ${ }^{2}$ Advanced Technology Institute, Nankai University, Tianjin 300071, China.

We present a novel scanning focused refractive-index microscopy (SFRIM) technique to obtain the refractive index (RI) profiles of objects. The method uses a focused laser as the light source, and combines the derivative total reflection method (DTRM), projection magnification, and scanning technique together. SFRIM is able to determine RIs with an accuracy of 0.002 , and the central spatial resolution achieved is $1 \mu \mathrm{m}$, which is smaller than the size of the focal spot. The results of measurements carried out on cedar oil and a gradient-refractive-index (GRIN) lens agree well with theoretical expectations, verifying the accuracy of SFRIM. Furthermore, using SFRIM, to the best of our knowledge we have extracted for the first time the RI profile of a periodically modulated photosensitive gelatin sample. SFRIM is the first RI profile-resolved reflected light microscopy technique that can be applied to scattering and absorbing samples. SFRIM enables the possibility of performing RI profile measurements in a variety of applications, including optical waveguides, photosensitive materials and devices, photorefractive effect studies, and RI imaging in biomedical fields.

$\mathrm{n}$ recent years, materials and devices with spatial refractive-index (RI) distributions that demonstrate characteristic functionalities have drawn extensive attention in a wide range of fields. Invisible cloaking using a properly designed RI profile is a topic of considerable interest, and the feasibility of these metamaterials is determined by their actual RI profile ${ }^{1-3}$. Waveguides, particularly optical fibers, are an important transmission medium that is widely used in research labs and daily life. The RI profile of a waveguide is an indispensable index in the assessment of their quality and performance ${ }^{4-6}$. Based on the detailed RI profile of these materials and devices mentioned above, researchers can obtain more direct and reliable information that can assist in their related research. The RI profile of bio-tissues and cells are traditionally and widely considered in the biomedical field $^{7-11}$. In contrast, experimental RI profiles of devices based on photosensitive materials ${ }^{12-14}$, which play an important role in information optics, are rarely reported. Thus far, a large number of RI profile measurement technologies have been developed. The refracted near-field (RNF) method, first demonstrated by Stewart ${ }^{15}$ in 1977 , is widely used at present, having undergone many years of development ${ }^{16,17}$. Although RNF provides the basis for the design of many commercial index-profiling instruments, it requires critical calibration to obtain an accurate measurement of the change in the RI. Many other techniques, such as quantitative phase microscopy $(\mathrm{QPM})^{18,19}$, differential interference contrast (DIC) microscopy ${ }^{20}$, scanning near-field microscopy $(\mathrm{SNOM})^{21}$, atomic force microscopy $(\mathrm{AFM})^{22}$, digital holographic microscopy $(\mathrm{DHM})^{23}$ and differential near-field scanning optical microscopy ${ }^{24}$, are described and can be applied in certain fields. The total internal reflection (TIR) method $^{25-29}$ plays an important role in RI measurements and has been widely adopted. Its spatial resolution is rather low because of its dependence on the commonly millimeter-level spot size of the beam. The low spatial resolution limits its application for the measurement of spatially resolved RI profile. Most traditional TIR methods must measure the angle-dependent reflectance curve, from which the base average RI of the illuminated area $\left(\mathrm{mm}^{2}\right.$ level) is obtained. In addition, the acquisition of each RI requires a series of data to be collected for rotations of the sample stage. However, the TIR method has the advantages of being both non-destructive and accurate. The excellent TIR method has advantages in RI measurement, and is a potential technique waiting to be applied in RI profile measurement.

In this study, we propose a novel scanning focused refractive-index microscopy (SFRIM) technique for quantitatively measuring RI profiles. The central spatial resolution of SFRIM is better than $1 \mu \mathrm{m}$, which is smaller than the size of the focal spot; and the RI accuracy is 0.002 . With a focused laser serving as the light source, SFRIM combines the derivative total reflection method (DTRM), projection magnification and the scanning technique to 


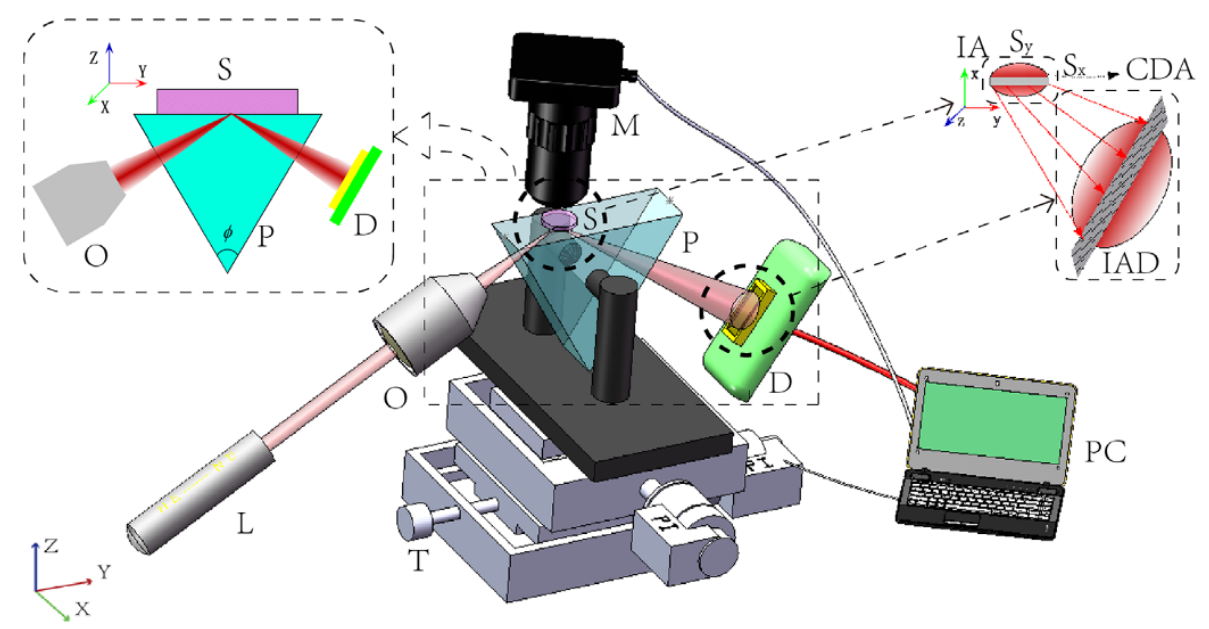

Figure 1 Structure diagram of SFRIM. L is a He-Ne laser, $\mathrm{O}$ is the long work distance objective lens, $\mathrm{S}$ is the sample, $\mathrm{P}$ is the prism, $\mathrm{D}$ is the detector, and $\mathrm{T}$ is the translation stage. $\phi$ is the apex angle of the prism. $X Y$-plane is horizontal. Central beam of the collimated beam is in YZ-plane, and the prismsample interface is parallel to the $X Y$-plane. The collimated beam out from the laser is focused on the prism-sample interface by the objective lens. Intensity of the reflected light is recorded by linear detector. The computer receives and analyzes data from the detector and controls movement of the translation stages. The enlarged diagram shows the relationship between the detected area and the illuminated spot. IAD is the imager area of the detector. $I A$ is the illuminated area on the sample and CDA is the correspondingly detected area. The spatial resolution of SFRIM is defined as the length on each direction of the detected area, which is $S_{x}$ along $X$-axis, and $S_{y}$ along Y-axis.

obtain the RI profile of objects. Based on the TIR method, using a focused laser beam incident on a sample surface, the RI profile of the sample can be obtained by successive scanning as the focal spot of the beam moves across the sample surface. The spatial resolution of SFRIM is considerably enhanced, and the SFRIM technique also avoids the necessity of time-consuming rotation in RI measurements. Moreover, as a TIR-based technology, SFRIM has the advantages of being non-destructive and accurate. In this work, measurements carried out on cedar oil, a GRIN lens, and photosensitive gelatin were used to experimentally verify the high spatial resolution and accuracy of SFRIM. This technique exhibits several appealing advantages, such as non-destructive, repeatable, and label-free measurement and ease of handling for a wide variety of substances, which makes SFRIM a promising approach in the field of RI profile measurement.

Microscopy can be simply classified into transmission-type and reflection-type. Current RI profile-resolved technologies, such as QPM, DHC and DIC microscopy, detect the transmitted light from the sample. To the best of our knowledge, SFRIM is the first RI profile-resolved reflecting microscopy that can be applied to scattering and absorbing samples, and is therefore supplementary to the existing RI profile microtechniques. Specimens for transmitted light microscopies are usually required to be transparent or semi-transparent and cut into slices, while SFRIM can be applied to thick and non-transparent samples.

Although TIR is used in SFRIM, surface plasmon resonance (SPR) sensors and total internal reflection microscopy (TIRF), there are many differences between these techniques. TIRF does not detect the TIR light and therefore it is not applied in the RI measurement. SPR sensors are highly sensitive to the RI change by detecting the intensity of optical wave near the resonance, while SFRIM records the reflectance curve and obtains RI values by determining critical angles. Moreover, SPR is not an RI profile-resolved technique. As the first RI profile-resolved reflected light microscopy, SFRIM is unique compared with the other TIR-based techniques.

\section{Results}

A schematic diagram of a SFRIM system is shown in Fig. 1. A He-Ne laser (HN4.0P, Newopto Technology Co.,Ltd, China) with a wavelength of $\lambda=632.8 \mathrm{~nm}$ serves as the light source, and the laser beam is focused with a $50 \times$ long-working-distance objective lens (WJ1050, focal length $4 \mathrm{~cm}$, working distance $20.5 \mathrm{~mm}$, N.A. 0.42. Beijing PaiVeiDi Technology Co.,Ltd, China). A sample attached to an equilateral prism is fixed on a pair of translation stages (M126, Physik Instrumente, Germany), and the focal spot lies on the horizontal interface. The translation stages can move along the $X$-axis or the $Y$-axis; therefore the sample together with the prism moves horizontally. The central axis of the focused beam is perpendicular to the airprism interface. A one-dimensional linear detector (Hamamatsu S3903-512Q) parallel to the prism-air interface is used to receive the reflected light. The polarization of the incident light is transverse-magnetic (TM) wave. Because the samples measured in this paper are isotropic, similar RI results would be obtained if the incidence light was transverse-electric (TE). When the sample is anisotropic, more consideration should be paid to the different polarization of incident light.

Because the diameter of the reflected light on the detection plane is wider than that of the detector, only a portion of the reflected light is detected. For each detected spot, the measurement time is only approximately $10 \mathrm{~ms}$, which is primarily determined by the response time of the detector. A computer controls the motorized stage and automated data collection, while the RI profile is calculated using MATLAB, making the SFRIM measurement simple and efficient. Because a large saturation exposure leads to a high signal-to-noise ratio and guarantees accurate measurement, we used an n-type metal-oxide-semiconductor (NMOS) detector with a high saturation exposure.

Figure 2 shows the propagation of the light through the SFRIM system. The angle-dependent reflectance curve $I_{r}$ can be calculated using:

$$
I_{r}=\left(I_{s}-I_{b}\right) /\left(I_{0}-I_{b}\right)
$$

where $I_{b}$ is the intensity distribution of the background light in the absence of an incident laser, and $I_{0}$ and $I_{s}$ are the reflected light distributions of the reference sample and target sample, respectively (Fig. 3(a)). We use air $\left(n_{\text {air }}=1.0003\right)$ as the reference sample. The incident angle of the focused beam should be varied across a range that covers the critical angle $\theta_{c}$ at the sample-prism interface, and the angle-dependent reflectance curve can be directly recorded by the detector. The critical angle $\theta_{c}$ is located at the peak of the first deriv- 


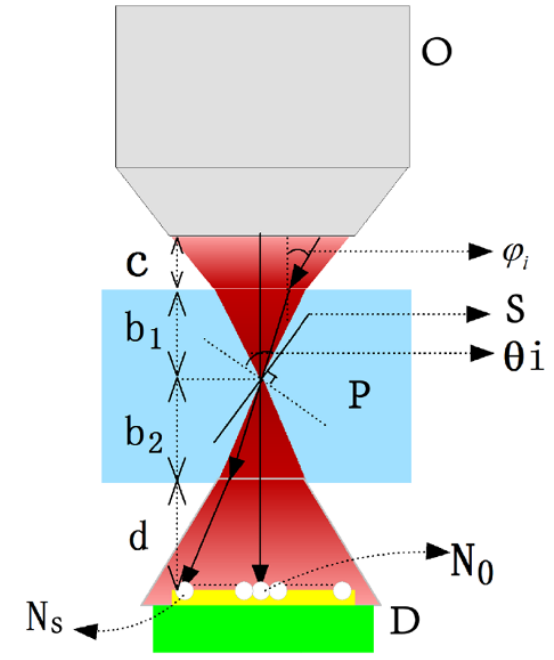

Figure $2 \mid$ Propagation of the light through the SFRIM system. $c$ is the distance between the objective and the prism. $d$ is the distance between the prism and the detector. $b_{1}$ and $b_{2}$ are the distances from the focal spot to the air-prism and prism-air interface, respectively. Propagation direction of the central beam of the collimated beam is along the $j$-axis and illuminate pixel $N_{0} . \varphi_{i}$ and $\theta_{i}$ are incident angle of the light that illuminated pixel $N_{i}$ on the air-prism interface and prism-sample interface, respectively.

ative of the reflectance curve ${ }^{28}$ (see Fig. 3(b)). The RI of the sample $n_{s}$ can then be calculated using Snell's law:

$$
n_{s}=n_{p} \sin \theta_{c}
$$

where $n_{p}$ is the RI of the prism. By successively changing the position of the focal spot during scanning, the measurement of the RI profile is obtained.
The detector only records the relationship between the light intensity and the pixel coordinate. The value of $\theta_{c}$ can be calculated using (see Supplementary theory):

$$
b \tan \left(\theta_{c}-\phi\right)+a \frac{n_{p} \sin \left(\theta_{c}-\phi\right) / n_{\text {air }}}{\sqrt{1-\left[n_{p} \sin \left(\theta_{c}-\phi\right) / n_{\text {air }}\right]^{2}}}=\gamma\left(N_{s}-N_{0}\right)
$$

where $N_{0}$ and $N_{s}$ denote the pixel coordinate corresponding to the center of the incident beam, and light beam with an incident angle $\theta_{c}$, respectively. Additionally, $\gamma$ is the distance between adjacent pixels; $\phi$ $=60^{\circ}$ is the prism apex angle, which has an angle tolerance of $1^{\prime} ; b_{1}$ and $b_{2}$ are the distance between the focal spot and the air-prism interface for the incident and reflected light, respectively; $b$ is the altitude of the ZF4 prism $\left(n_{p}=1.723\right.$; side length tolerance $=$ $0.05 \mathrm{~mm}$ ) that satisfies $b=b_{1}+b_{2}$; and $a$ is a constant distance parameter. Calibration of the system is performed using two standard samples with known RIs to calculate $a$ and $N_{0}$.

The systematic error can be theoretically calculated according to the well-known error-propagation formula. The maximum systematic error of the RI is calculated to be less than 0.002 and gradually reaches its minimum when $\theta_{c} \rightarrow \beta\left(N_{s} \rightarrow N_{0}\right)$ (see Supplementary theory). The use of the derivative method enables the simply-structured SFRIM to be applied to both transparent samples and non-transparent samples with weak absorbing or scattering ${ }^{28}$, which has shown great potential to be a widely used microscopy technique.

To experimentally verify the theoretical accuracy, a measurement was performed using cedar oil at room temperature. The RI of cedar oil is $1.5150 \pm 0.0002$ according to the data provided in the product manual. Glycol $(n=1.4318)$ and glycerol $(n=1.4746)$ were chosen as standard samples for the system calibration. Using equation (3), the constant parameters $a$ and $N_{0}$ were calculated to be $a=$ $0.1158 \mathrm{~cm}, N_{0}=251.5463$. Fig. 3(c) shows the normalized reflected intensity distributions of glycol, glycerol, cedar oil, and the background. The reflectance curves and derivatives of the reflectance
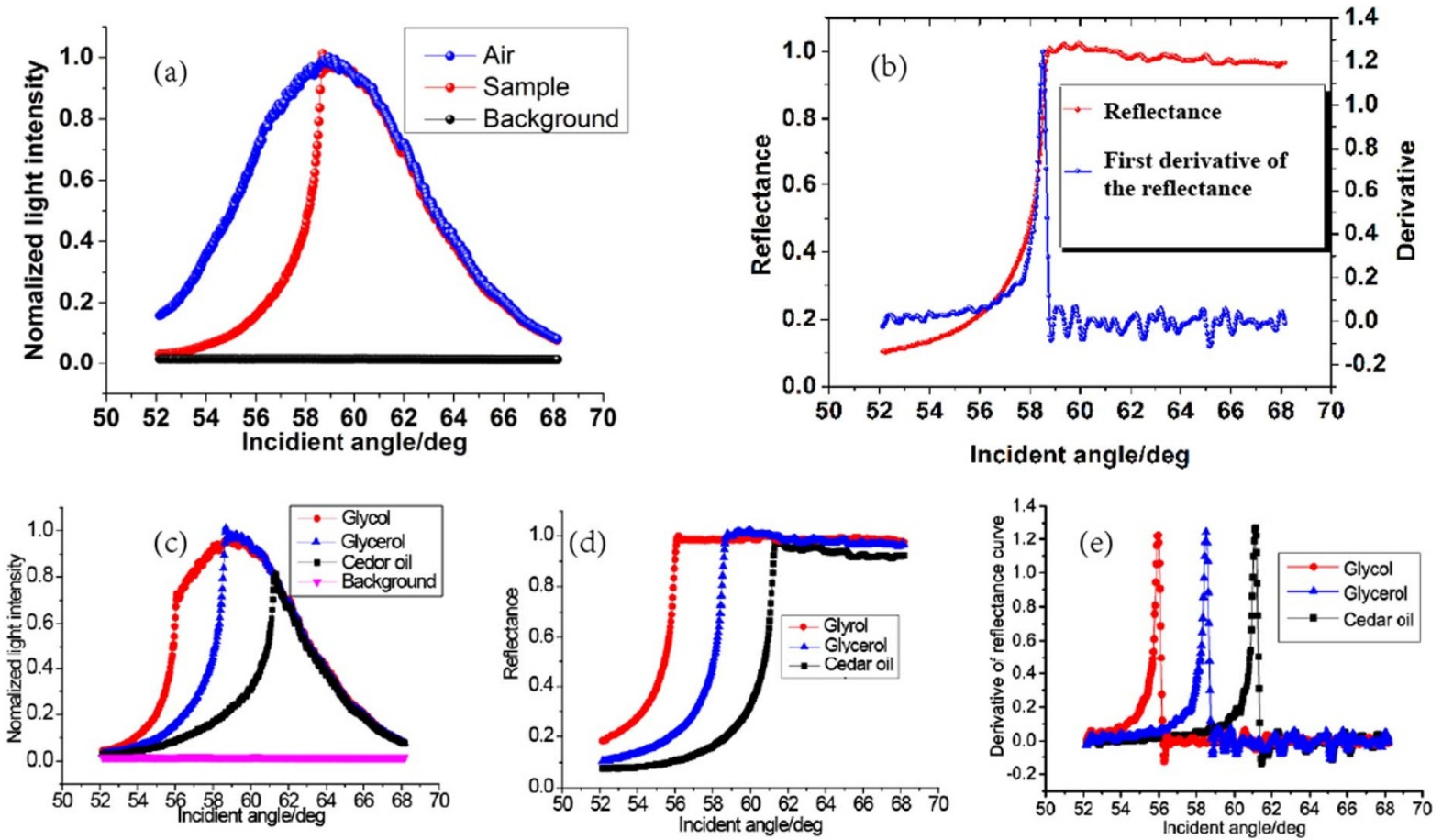

Figure 3 | (a) Normalized intensity of reflected light curves for air, sample and background. The curves are normalized with respect to the maximum value of the intensity of the air. (b) Reflectance curve of sample and its first derivative curve. (c) Normalized reflected intensity of glycol, glycerol, cedar oil and background. (d) Distribution of reflectance curves for glycol, glycerol and cedar oil. (e) First derivative of reflectance curves for glycol, glycerol and cedar oil. It shows that information of reflected light with $\theta_{i} \in\left(\theta_{c}-1^{\circ}, \theta_{c}+1^{\circ}\right)$ is sufficient to confirm the peak of first derivative of reflectance-angle curve. 
curves of each sample are shown in Figs. 3(d) and (e), respectively. The RI of cedar oil was measured ten times to calculate the random error and the standard deviation, which was calculated to be 1.5148 \pm 0.0001 .

To demonstrate the accuracy of SFRIM in an RI profile measurement, a GRIN lens (Thorlabs, GRIN 2906) was chosen as a typical sample with a known theoretical RI profile. According to the product manual, the theoretical expression for the RI profile of the GRIN lens is $n_{r}=1.6073 \times\left[1-\left(r \times 0.339 \mathrm{~mm}^{-1}\right)^{2} / 2\right]$, where $r$ is the radial distance. We scanned a few lines near the center of the sample to measure the RI profile. Measurements were made in intervals of $1 \mu m$ along the $X$-axis and $45 \mu m$ along the $Y$-axis. The coordinate $Y=0 \mu m$ indicates the central line. The 2-D RI profile of the GRIN lens is shown in Fig. 4 (a). The theoretical values and the measured results at a fixed $X$ or $Y$ coordinate are compared in Fig. 4 (b) and (c). There is fairly close agreement between the theoretical values and the measured RI profile.

Furthermore, in addition to traditional uniform materials and optical waveguide devices, SFRIM opens up the possibility to measure RI profiles of photosensitive materials. Without the modulation and development process, the RI values for doped and undoped gelatin were previously measured by SFRIM to be $1.541 \pm 0.002$ and $1.551 \pm 0.002$, respectively. Figure 5 shows the experimental RI profile of a photosensitive gelatin sample with a modulation period of $40 \mu \mathrm{m}$. The measured intervals were $1 \mu \mathrm{m}$ along the $X$-axis and $15 \mu \mathrm{m}$ along the $Y$-axis. A noticeable decrease was observed after the development process, and the RI of the sample was periodically reduced to $1.413 \sim 1.435$. As observed from the $2-\mathrm{D}$ scan of the RI profile and the measured results at a fixed $Y$ coordinate in Fig. 5 (b), the period of the RI is in close agreement with the modulated period, while the valleys and peaks of the RI values remained nearly the same (Figs. 5(c) and (d)).

\section{Discussion}

As an RI profile-resolved technique, the accuracy of SFRIM is important. Potential sources of systematic error include errors in the tolerance of the prism (mainly involving the angle tolerance and the side-length tolerance), background, dark current of the detector, fluctuations in the laser intensity, mechanical errors, and calculation error of the derivative method. The influence of the background is eliminated by equation (1). Although fluctuations in the laser power affect the integral light intensity of the reflectance curve, the peak position of the first derivative of the curve is unaffected. The maximum dark current of the detector is $0.08 \mathrm{pA}$ and contributes little to the total error. Mechanical error that arises during the scanning process only affects the measured light distribution and does not alter the RI accuracy.

Therefore the accuracy of SFRIM is primarily affected by the tolerance of the prism and the calculation error of the derivative method. The tolerances of the apex angle and the side length of a given prism are constant and are provided in the product manuals. If these parameters are determined more precisely, their effects on the total experimental error can be lower. The calculated values of $N_{s}$ and $N_{0}$ are integers; therefore, the value of the measured RI is discrete, which is the main source of the calculation error of the derivative method. Because the errors of both $N_{s}$ and $N_{0}$ are within \pm 0.5 , the total error of $N_{0}-N_{s}$ is \pm 1 , which corresponds to an RI error of approximately $\pm 4 \times 10^{-4}$. Therefore, the accuracy of SFRIM can be improved by using a detector with smaller a pixel size.

SFRIM offers unique advantages over other traditional TIR techniques for measuring RI profiles with high spatial resolution, which in this case is much smaller than the size of the focal spot. The reflected light at the focal spot (infinitely close to the prism-sample interface) is projected and magnified onto the detection plane. The theoretical magnification factor $M_{t}$ is defined as

$$
M_{t}=w / w_{0}
$$

where $w$ is the radius of the reflected light spot on the detection plane and $w_{0}$ is the beam waist of the reflected light. During the propagation of the Gaussian beam from air into the prism, the position of the focal spot changes, but the waist size remains constant in the prism. Instead of measuring the waist in the prism, we measured it in the air and found that $w_{0} \approx 3.5 \mu \mathrm{m}$ (see Supplementary theory). If the detector is not far from the focal spot, the interference of the reflected light from adjacent areas can be ignored. The intensity distribution of the reflected light on the detection plane corresponds to that of the reflected light at the focal spot, which means the detected area is a portion of the reflected light (see the enlarged diagram in Fig. 1).
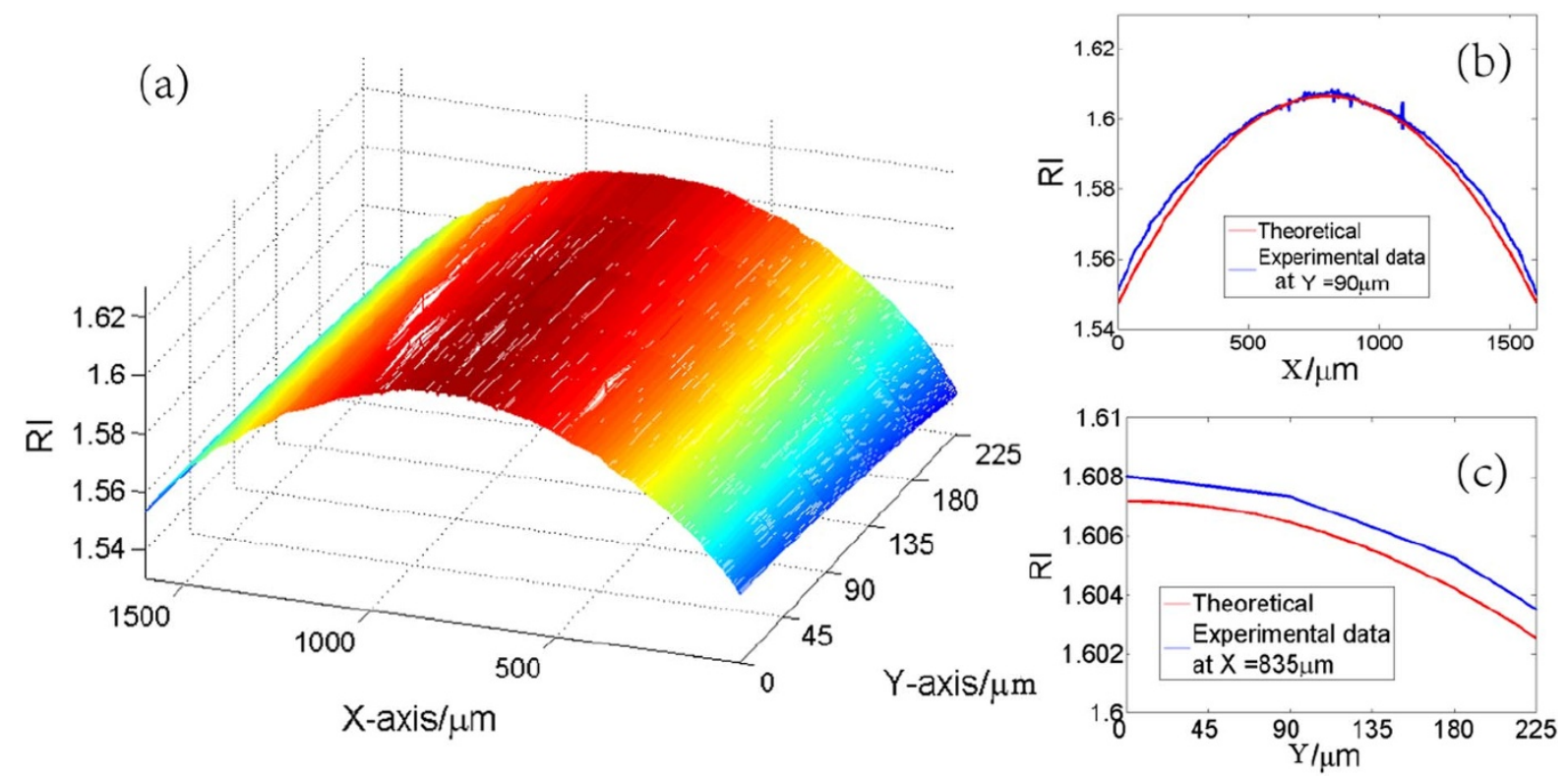

Figure $4 \mid$ (a) Central part of RI profile of a GRIN lens scanned by SFRIM. (b) Measured and theoretical RI profile of the GRIN lens along the $X$-axis at $Y=$ $90 \mu \mathrm{m}$. There is slight departure at the fringe area. Photo of the arc-shaped area is shown in supporting material. (c) Measured and theoretical RI profile along the $Y$-axis at $X=835 \mu \mathrm{m}$. The measured data departure from theoretical expectation is less than 0.001 . 


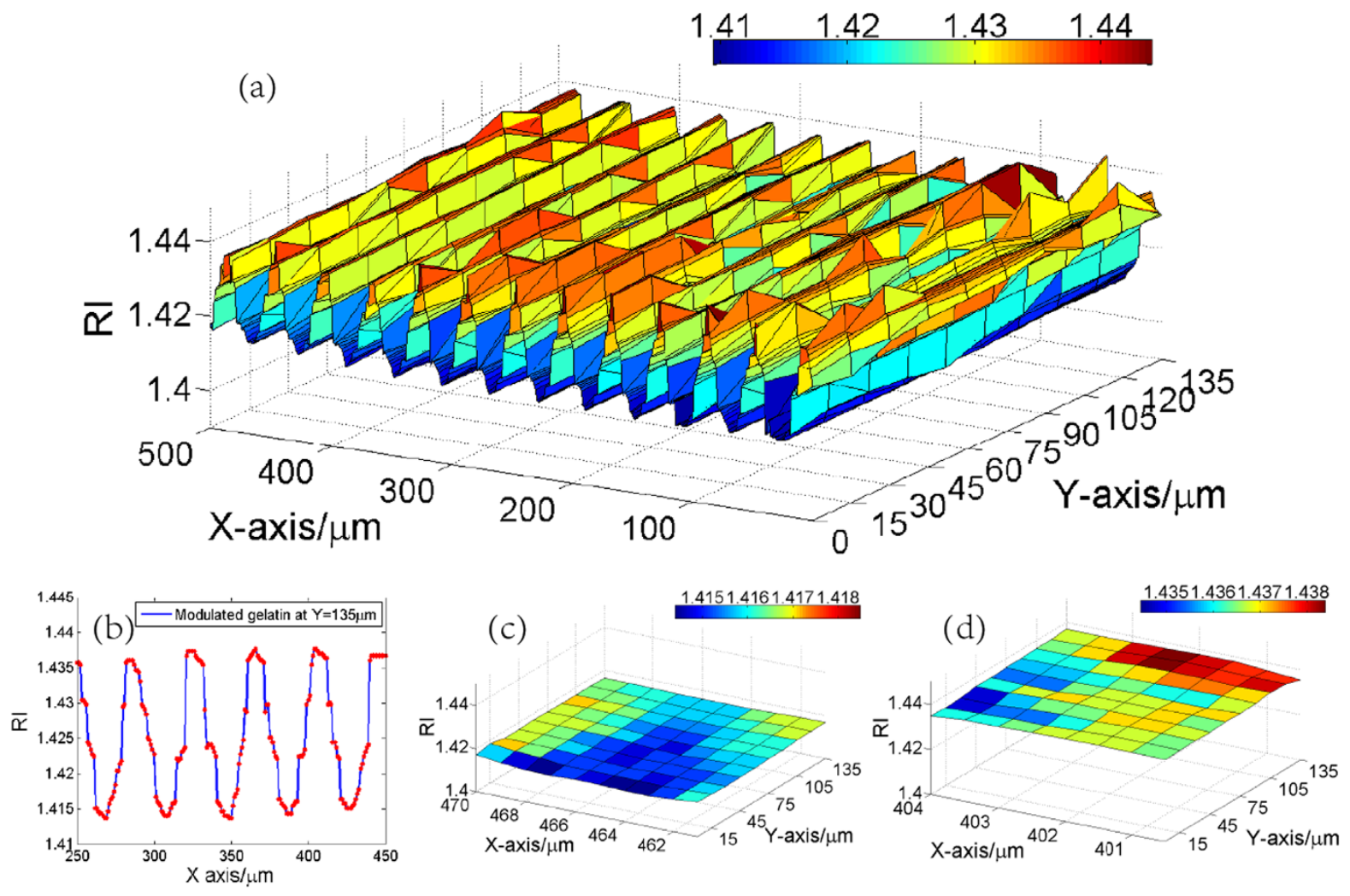

Figure $5 \mid$ (a) RI profile of the modulated gelatin. (b) Detailed RI profile of the modulated gelatin along the $X$-axis at $Y=135 \mu m, X \in[250,450] \mu m$. (c) RIs for the valley of the modulated gelatin. (d) RIs for the peak of the modulated gelatin.

Assuming that the center of the focal plane is the origin of the coordinate system and that the light propagates along the $j$-axis, the beam radius $w(j)$ is ${ }^{30}$ :

$$
w(j)=w_{0} \cdot \sqrt{1+\left(\frac{j \cdot \lambda}{\pi \cdot n_{\text {air }} \cdot w_{0}^{2}}\right)^{2}}
$$

On the detection plane, $j \approx 40 \mathrm{~mm}, w(j) \approx 2.3 \mathrm{~mm}$. By equation (4), the calculated $M_{t} \approx 658$. The experimental magnification factor $M_{e}$ was determined using a resolution plate with a period of $2 \mu \mathrm{m}$ and a duty cycle of $1: 1$. The image of the resolution plate that appeared on the detection plane was recorded. The width of the brightest light stripe in the center was approximately $650 \mu \mathrm{m}$ (see Supplementary Fig. S3 online), which means that $M_{e} \approx 650$.

The spatial resolution of SFRIM is defined as the length along each direction of the detected area, which is denoted as $S_{x}$ along $X$-axis, and $S_{y}$ along $Y$-axis. The width and the length of the detector are $q=$ $0.5 \mathrm{~mm}$ and $p=12.8 \mathrm{~mm}$, respectively. The diameter of the reflected light spot on the detection plane is $4.6 \mathrm{~mm}$, which is larger than $q$ and smaller than $p$ (the enlarged diagram in Fig. 1). Therefore, $S_{x}$ is calculated using:

$$
S_{x}=q / M_{e}
$$

and is found to be equal to $0.8 \mu \mathrm{m}$.

The spatial resolution along the $Y$-axis $S_{y}$ equals the length $l$ of the illuminated area on the sample. The illuminated area is the cross section of the Gaussian beam and the prism-sample interface. $S_{y}$ is calculated using:

$$
S_{y}=\left|j_{1}-j_{2}\right| / \cos \phi
$$

where $j_{1}$ and $j_{2}$ are the roots of the equation set:

$$
\left\{\begin{array}{l}
\left(\frac{w(j)}{w_{0}}\right)^{2}-\left(\frac{j \cdot \lambda}{\pi \cdot n_{p} \cdot w_{0}^{2}}\right)^{2}=1 \\
w(j)=j \cdot \cot \phi
\end{array}\right.
$$

The calculated value of $S_{y}$ is found to be $14.0 \mu \mathrm{m}$.

The information obtained with reflected light in the range $\theta_{i} \in\left(\theta_{c}\right.$ $\left.-1^{\circ}, \theta_{c}+1^{\circ}\right)$ is sufficient to obtain the RI. This indicates that if the sample is previously measured to roughly determine the range of $\theta_{c}$, a diaphragm can be added in front of the detector to ensure that the incident angle of the transmitted light covers $\theta_{c}$. This procedure is equivalent to the shortening of $l$ and improves $S_{y}$ to approximately $3.8 \mu \mathrm{m}$. The spatial resolution along the $Z$-axis can be defined as the depth of penetration of the evanescent wave ${ }^{31}$ :

$$
d=\frac{\lambda}{4 \pi \sqrt{n_{p}^{2} \sin ^{2} \theta_{i}-n_{s}^{2}}}
$$

which is on the order of the wavelength of light used ${ }^{32}$.

When the translation stage moves along the $Y$-axis, the size of the illuminated area increases hyperbolically and both $S_{x}$ and $S_{y}$ decreases slightly. When the translation stage moves $250 \mu \mathrm{m}$ along the Y-axis, $S_{x}$ and $S_{y}$ worsens to $1.8 \mu \mathrm{m}$ and $7.8 \mu \mathrm{m}$, respectively, which indicates that both $S_{x}$ and $S_{y}$ change slightly over a fairly large range. Below the diffraction limit, the $S_{x}$ and $S_{y}$ of SFRIM can be improved by several methods, including increasing the distance between the objective lens and the output mirror of the laser, and increasing the distance between the detector and the focal spot.

Current RI profile-resolved techniques, such QPM, DHM and DIC microscopy, commonly have diffraction-limited $(\sim 1 \mu \mathrm{m})$ transverse resolution, RI accuracies of 0.01 to $0.0002^{20,23}$. The spatial resolution of SFRIM is similar to that of the techniques mentioned above and the RI accuracy of SFRIM is at an intermediate level compared with these transmission-based microscopies. Moreover, SFRIM has unique advantages over current techniques because it can be widely 
applied to thick and non-transparent samples with scattering and absorption effect. The RI sensitivity of SFRIM is defined as $d \theta / d n$ or $d N / d n$, which are approximately $104.2 \mathrm{deg} / \mathrm{RIU}$ or $3472.2 \mathrm{RIU}^{-1}$, respectively, for our apparatus. This figure of merit is reported to be $5.7 \times 10^{4} \mathrm{~nm} /$ RIUfor an SPR sensor ${ }^{33}$. However, the SPR technique has not been used for RI profile measurements. Because the minimum distinguishable angle for SFRIM is $0.03^{\circ}$, the minimum distinguishable RI change is approximately $2.88 \times 10^{-4}$.

For the cedar oil and modulated gelatin samples, which were made on the surface of the prism, it is difficult to only move the sample. Theoretically, it is better to move the sample alone to avoid the variation of the illuminated area. However, flatness of the prism and the sample are not ideal. The sample can't move absolutely horizontal. For solid samples, the thickness change of the matching liquid during movement will affect the results. Besides, when the prism moves together with the sample in the Y-axis, both $S_{x}$ and $S_{y}$ slightly change over a fairly large area. Therefore, it is unavoidable that the sample and prism move together in our setup.

The RI profile of the GRIN lens is inhomogeneous and can be considered to be continuously varying along the $Y$-axis of the detected area. Therefore, the measured RI is the mean RI over each detected area. It is notable that the shift of the measured RI is regular to a certain extent rather than random spread along the radial direction (see Fig. 4(b)). It is therefore unlikely that the deviation from the theoretical profile is caused by random measurement errors. Figure 4(c) shows the deviation between the experimental and theoretical profile in the central part of the GRIN lens, which is clearly less than 0.001 . The fringe area is polished to be arc-shaped, which is unavoidable during production; therefore, the slight departure of the measurement from theory is most likely attributed to the geometrical irregularities of the GRIN lens at the fringe area. The shift of the experimental values to larger values than compared with the central part can be attributed to the non-uniform thickness of the matchingliquid between the GRIN lens and the prism, which is thicker near the fringe area (see Supplementary Fig. S4 online). Several fluctuations are observed in the central area (see Fig. 4(b)), which are most likely caused by scratches on the prism or the end face of the GRIN lens. The effect of these scratches is similar to the geometric irregularities at the fringe area. Apart from the influence of geometric irregularities, the effect of the angle and side-length tolerances introduces a constant error, so all of the measured data exhibited a tendency to increase unidirectionally. This finding serves as evidence that the accuracy of SFRIM can be further improved. Additionally, because the GRIN lens is placed on the prism directly, no polishing or other destructive manipulation is needed, making SFRIM non-destructive and repeatable. By adding an index-matching liquid, SFRIM is capable of extracting the RI profile of a solid sample as well as a liquid sample with high accuracy.

The RI of the periodic modulated gelatin also varies continuously along the $X$-axis, so the results are again the mean RI over each detected area. As shown in Figs. 5 (a) and (b), there are slight irregularities in the periodically modulated RI profile. Gelatin was poured directly onto the prism surface to minimize the effect of geometric irregularities. The slight irregularities in the curve are likely due to dust-based non-uniformities mixed into the gelatin solution or by laser speckles illuminating irregular spots during the exposure process. The measured period is identical to the period of the mask plate. To the best of our knowledge, this is the first experimentally measured RI profile of a modulated photosensitive gelatin. The measured data provide direct and reliable information on which to base further studies and theoretical analyses of photosensitive gelatin and will help facilitate the studies of photonic crystals ${ }^{34}$ and photonic bandedge lasing ${ }^{35}$. The quantitative RI profile of other photosensitive materials can also be mapped using SFRIM.

In conclusion, we have developed the first RI profile-resolved reflecting microscopy that can be applied to scattering and absorbing samples with high spatial resolution and accuracy. We have demonstrated that this setup has a spatial resolution of $1 \mu \mathrm{m}$ and an RI measurement accuracy of 0.002 . Experimental values of both liquid and solid samples have been presented, which demonstrate both the feasibility and the advantages of the proposed RI profile microscopy technique. Moreover, for the first time we have measured the RI profile of a periodically modulated gelatin. SFRIM offers advantages such as non-destructive, repeatable measurements and ease of handling. It can be applied in many fields, including measurements of the RI profile of optical waveguides and photosensitive materials, studies of the characteristics of photorefractive materials, and label-free, non-destructive in vivo RI imaging in biological applications.

\section{Methods}

Derivative total reflection method. The refractive index (RI) values are obtained by determining the critical angle, beyond which total internal reflection (TIR) will occur. There is a sudden change in the reflected light intensity at the critical angle for a nonabsorbing sample. However, most samples are weakly absorbing and/or induce scattering, which makes the critical angle difficult to determine visually using the reflectance curve.

Based on the Fresnel equation, the reflectance $R$ for s-polarized light is:

$$
R=\frac{\left(n_{p} \cos \theta-\mu_{2}\right)^{2}+v_{2}^{2}}{\left(n_{p} \cos \theta+\mu_{2}\right)^{2}+v_{2}^{2}}
$$

where the parameters $\mu_{2}$ and $v_{2}$ are the intermediate variables

$$
\begin{aligned}
& 2 \mu_{2}^{2}=n_{s}^{2}\left(1-\chi^{2}\right)-n_{p}^{2} \sin ^{2} \theta+\sqrt{\left[n_{s}^{2}\left(1-\chi^{2}\right)-n_{p}^{2} \sin ^{2} \theta\right]^{2}+4 n_{s}^{4} \chi^{2}} \\
& 2 v_{2}^{2}=-\left[n_{s}^{2}\left(1-\chi^{2}\right)-n_{p}^{2} \sin ^{2} \theta\right]+\sqrt{\left[n_{s}^{2}\left(1-\chi^{2}\right)-n_{p}^{2} \sin ^{2} \theta\right]^{2}+4 n_{s}^{4} \chi^{2}}
\end{aligned}
$$

$x$ is the extinction coefficient, $x=\left(\mu_{s}+\mu_{a}\right) \lambda / 4 \pi$, and $\mu_{s}$ and $\mu_{a}$ are the scattering coefficient and the absorption coefficient, respectively. The critical angle is located at the maximum of the first derivative of equation $(10)^{28}$. Because equation (10) contains the scattering coefficient and the absorption coefficient, DTRM and therefore SFRIM are applicable to scattering and absorbing samples. The above result is also suitable for p-polarized light.

Preparation of cedar oil. The samples chosen for traditional RI measurement methods are typically uniform liquid materials, such as $\mathrm{NaCl}$ or sucrose solutions. The prism-sample interface was oriented horizontally in our setup, and the cleanup procedure can be easily performed with the prism fixed on the translation stage. One drop (approximately $0.05 \mathrm{ml}$ ) of cedar oil surrounding the beam spot on the prism is sufficient for the measurement, thus avoiding the potential mechanical error arising from replacement of the prism.

Preparation of a gradient-refractive-index (GRIN) lens. The index-matching liquid used in this study was manufactured by Cargille Laboratories and had an RI of 1.7320 \pm 0.0002 . It was added to the interface of the prism and the GRIN lens, and the end face of the latter was carefully cleaned beforehand. The weight of the GRIN lens was approximately $0.04 \mathrm{~g}$, and the microscope scans in a smooth manner; therefore, no relative movement occurred during the measurement process.

Preparation of the photosensitive gelatin. The preparation process for the photosensitive gelatin can be mainly divided into four steps: (1) preparation of the formulated solution, (2) coating the solution of $300 \mu l$ onto the prism and dry, (3) exposure, (4) development process based on Ying's procedure ${ }^{36}$. Our exposure process differs from Ying's in that the mask plate with a period of $40 \mu \mathrm{m}$ and a duty cycle of $1: 1$ is placed closely in front of the coated gelatin film. The exposure time is $30 \mathrm{~s}$ using a laser wavelength of $488 \mu \mathrm{m}$ and an energy density of $0.611 \mathrm{~J} / \mathrm{mm}^{2}$.

1. Ergin, T., Stenger, N., Brenner, P., Pendry, J. B. \& Wegener, M. Three-dimensional invisibility cloak at optical wavelengths. Science 328, 337-339 (2010).

2. Lie, R. et al. Broadband ground-plane cloak. Science 323, 366-369 (2009).

3. Leonhardt, U. Optical conformal mapping. Science 312, 1777-1780 (2006).

4. Yang, Y. et al. Optofluidic waveguide as a transformation optics device for lightwave bending and manipulation. nat. commun. 3, 651 (2012).

5. Sazio, P. J. A. et al. Microstructured optical fibers as high-pressure microfluidic reactors. Science 311, 1583-1586 (2006).

6. Koike, Y. \& Asai, M. The future of plastic optical fiber. NPG ASIA MATER 1, 22-28 (2009).

7. Jeong, K. H., Kim, J. \& Lee, L. P. Biologically inspired artificial compound eyes. Science 312, 557-561 (2006).

8. Phillips, K. G., Jacques, S. L. \& McCarty, O. J. T. Measurement of Single Cell Refractive Index, Dry Mass, Volume, and Density Using a Transillumination Microscope. phys. rev. lett. 109, 118105 (2012). 
9. Wang, Z., Tangella, K., Balla, A. \& Popescu, G. Tissue refractive index as marker of disease. J. biomed. Opt. 16, 116017 (2011).

10. Verma, Y., Rao, K. D., Suresh, M. K., Patel, H. S. \& Gupta, P. K. Measurement of gradient refractive index profile of crystalline lens of fisheye in vivo using optical coherence tomography. Applied Physics B 87, 607-610 (2007).

11. Nilsson, D. E., Gislén, L., Coates, M. M., Skogh, C. \& Garm, A. Advanced optics in a jellyfish eye. Nature 435, 201-205 (2005).

12. Doshi, D. A. et al. Optically Defined Multifunctional Patterning of Photosensitive Thin-Film Silica Mesophases. Science 290, 107 (2000).

13. Grotjohann, T. et al. Diffraction-unlimited all-opticalimaging and writing with a photochromic GFP. Nature 478, 204-208 (2011).

14. Ishii, N., Kato, T. \& Abe, J. A real-time dynamic holographic material using a fast photochromic molecule. Sci. Rep. 2, 819 (2012).

15. Stewart, W. J. A new technique for measuring the refractive index profiles of graded optical fibers. Technical Digest 1006, IOOC, Tokyo. IECE, Tokyo. (1977, July, 18-20).

16. Raine, K. W., Baines, J. G. N. \& Putland, D. E. Refractive Index Profiling-State of the Art. J. lightwave technol. 7, 1162-1169 (1989).

17. Oberson, P., Gisin, B., Huttner, B. \& Gisin, N. Refracted near-field measurements of refractive index and geometry of silica-on-silicon integrated optical waveguides. Appl. Optics. 37, 7268-7272 (1998).

18. Roberts, A., Ampem-Lassen, E., Barty, A. \& Nugent, K. A. Refractive-index profiling of optical fibers with axial symmetry by use of quantitative phase microscopy. Opt. Lett. 27, 2061-2063 (2002).

19. Ampem-Lassen, E., Sidiroglou, F., Peng, J. L., Huntington, S. T. \& Roberts, A. Index mapping for fibers with symmetric and asymmetric refractive index profiles. opt. express. 16, 10912-10917 (2008).

20. Dragomir, N. M. et al. Refractive index profiling of optical fibers using differential interference contrast microscopy. photonic tech. l. 17, 2149-2151 (2005).

21. Radko, I. P., Volkov, V. S., Bozhevolnyi, S. I., Henningsen, J. \& Pedersen, J. Nearfield mapping of surface refractive-index distributions. Laser Phys. Lett. 2, 440-444 (2005).

22. Pace, P., Huntington, S. T., Lyytikäinen, K., Roberts, A. \& Love, J. D. Refractive index profiles of Ge-doped optical fibers with nanometer spatial resolution using atomic force microscopy. Opt. express. 12, 1452-1457 (2004).

23. Charrière, F. et al Cell refractive index tomography by digital holographic microscopy. Opt. Lett. 31, 178-180 (2006).

24. Tsai, W. S., Wang, W. S. \& Wei, P. K. Two-dimensional refractive index profiling by using differential near-field scanning optical microscopy. Appl. Phys. Lett. 91, 061123 (2007)

25. Bolin, F. P., Preuss, L. E., Taylor, R. C. \& Ference, R. J. Refractive index of some mammalian tissues using a fiber optic cladding method. Appl. Optics. 28, 2297-2303 (1989).

26. Ding, H. f., Lu, J. Q., Wooden, W. A., Kragel, P. J. \& Hu, X. H. Refractive indices of human skin tissues at eight wavelengths and estimated dispersion relations between 300 and 1600 nm. Phys. Med. Biol. 51, 1479-1489 (2006).

27. Sun, P. Determination of the anisotropy complex refractive indices of chicken tissues in vitro at $650 \mathrm{~nm}$. J. eur. Opt. soc-rapid. 5, 10030 (2010).

28. Zhang, C. P., Song, Q. W., Ku, C. Y., Gross, R. B. \& Birge, R. R. Determination of the refractive index of a bacteriorhodopsin film. Opt. Lett. 19, 1409-1411 (1994).
29. McClimans, M., LaPlante, C., Bonner, D. \& Bali, S. Real-time differential refractometry without interferometry at a sensitivity level of $10^{-6}$. Appl. Optics. 45, 6477-6486 (2006).

30. Self, S. A. Focusing of spherical Gaussian beams. Appl. Optics. 22, 658-661 (1983).

31. Mirabella, F. M. Internal Reflection Spectroscopy: Theory and Applications1st edn (Marcel Dekker, New York, 1993).

32. Born, M. \& Wolf, E. Principles of Optics 6th edn (Pergamon, New York, 1986).

33. Slavík, R. \& Homola, J. Ultrahigh resolution long range surface plasmon-based sensor. Sens. Actuators, B 123, 10-12 (2007)

34. Ren, Z., Zhai, T. R., Wang, Z. N., Zhou, J. \& Liu, D. H. Complete Band Gaps in the Visible Range Achieved by a Low-Refractive-Index Material. Adv Mater. 20, 2337-2340; DOI:10.1002/adma.200702698 (2008).

35. Kok, M. H. et al Lasing from dye-doped photonic crystals with graded layers in dichromate gelatin emulsions. Appl. Phys. Lett. 92, 151108 (2008).

36. Ying, C. F., Zhou, W. Y., Ye, Q., Zhang, X. L. \& Tian, J. G. Band-edge lasing in Rh6G-doped dichromated gelatin at different excitations. J. Opt. 12, 105101 (2010).

\section{Acknowledgments}

This research is supported by the Chinese National Key Basic Research Special Fund (Grant No.2011CB922003), Science and Technology Program of Tianjin (Grant No.11ZCKFSY00500).

\section{Author contributions}

The experiment were conceived, designed and carried out by T.Q.S., Q.Y. and Z.C.D., X.W.W. prepared the samples. T.Q.S., Q.Y., X.W.W. and J.C.M. contributed to the the interpretation of the data and developed analytical and computational theoretical tools. J.G.T., Q.Y., C.P.Z., W.Y.Z. and J.W. analyzed and interpreted the data. Q.Y., T.Q.S. and J.G.T. wrote the manuscript with the assistance of all other co-authors.

\section{Additional information}

Supplementary information accompanies this paper at http://www.nature.com/ scientificreports

Competing financial interests: The authors declare no competing financial interests.

How to cite this article: Sun, T.-Q. et al. Scanning focused refractive-index microscopy. Sci. Rep. 4, 5647; DOI:10.1038/srep05647 (2014).

This work is licensed under a Creative Commons Attribution-NonCommercialShareAlike 4.0 International License. The images or other third party material in this article are included in the article's Creative Commons license, unless indicated otherwise in the credit line; if the material is not included under the Creative Commons license, users will need to obtain permission from the license holder in order to reproduce the material. To view a copy of this license, visit http:/ creativecommons.org/licenses/by-nc-sa/4.0/ 